\title{
TweeProfiles3: visualization of spatio-temporal patterns on Twitter
}

\author{
André Maia ${ }^{1}$, Tiago Cunha ${ }^{1}$, Carlos Soares ${ }^{1}$, and Pedro Henriques Abreu ${ }^{2}$ \\ 1 Faculdade de Engenharia da Universidade do Porto, Porto, Portugal \\ \{ee08163,tiagodscunha,csoares\}@fe.up.pt \\ 2 Departamento de Engenharia Informática da Universidade de Coimbra \\ Coimbra, Portugal \\ pha@dei.uc.pt
}

\begin{abstract}
With the advent of social networking, a lot of user-specific, voluntarily provided data has been generated. Researchers and companies noticed the value that lied within those enormous amounts of data and developed algorithms and tools to extract patterns in order to act on them. TweeProfiles is an offline clustering tool that analyses tweets over multiple dimensions: spatial, temporal, content and social. This project was extended in TweeProfiles 2 by enabling the processing of real-time data. In this work, we developed a visualization tool suitable for data streaming, using multiple widgets to better represent all the information. The usefulness of the developed tool for journalism was evaluated based on a usability test, which despite its reduced number of participants yielded good results.
\end{abstract}

Keywords: Clustering, Information Visualization, Stream processing

\section{Introduction}

Online social networks present a variety of social media services which have achieved a huge importance in social life as well as in marketing strategies as they "have been regarded as a timely and cost-effective source of spatio-temporal information" [10]. One business which has been significantly affected is journalism. Social networks can be used to change the way journalists are able to take the pulse of the trending topics. Twitter is one of the top social networks, both in popularity and monthly active users (around 250 million). It is a starting point for our ongoing work because it is the most widely used microblogging application.

The first version of TweeProfiles [6] is focused on identifying profiles on data collected from Twitter. The data is processed over 4 dimensions (spatial, temporal, social and content) using Data Mining techniques. The tool enables the visualization of the results of the clustering algorithm. Since it lacked the ability to produce real-time visualizations of the evolution of the data stream, one extensions was developed: TweeProfiles2 [14]. It replaced the original batch clustering algorithm with a stream clustering algorithm, enabling the use of real-time 
data. However, the system was not fully implemented and still used static data to perform clustering.

Until now, all work performed was essentially technical and scientific, in order to design and prototype solutions to the challenges involved. For this reason, it has not yet been possible to evaluate the visualization platform in a real-world application. Our motivation for this work lies with the design of an extension for TweeProfiles2. Our most important contribution is the development of a visualization system with an interactive representation of the profiles. In addition, we also included connections to other platforms in order to enrich the profiles and ease the access to relevant information. We used services provided by SAPO Labs $^{3}$, which devote themselves to the extraction and analysis of several online data sources related to the Portuguese community. We also aim to evaluate this tool in a real-world application, as well as to integrate the previous process with a real-time data extraction platform named SocialBus ${ }^{4}$ [3]. We discuss this tool in more detail in Section 3.

This paper is organized as follow: Section 2 contains the State of the Art for the research areas of clustering and information visualization. Section 3 presents the developed system with the concepts and decisions for the data mining process and the visualization tool. Section 4 presents some results obtained from our analysis of this project. Finally, Section 5 lists our conclusions and tasks for future work.

\section{State of The Art}

\subsection{Clustering}

Data mining is the process of exploring large amounts of data with the goal of finding potentially interesting patterns [9]. One of its most well-known tasks is Clustering and it is defined as the process of grouping a set of data objects into multiple groups or clusters so that objects within a cluster have high similarity, but are very dissimilar to objects in other clusters [9]. Over the years, cluster techniques have been applied in multiple domains, e.g. profiling breast cancer patients to predict overall survival [1], helping to choose the best strategy to defeat a soccer opponent [2] among others. Since this project follows the work done in TweeProfiles2, one of its main tasks is to perform clustering on data collected from Twitter, specifically using Stream Clustering approaches. Stream Clustering is a research area that recently emerged to discover knowledge from large amounts of continuously generated data. In this context, several algorithms have been proposed to perform unsupervised learning [15]. Since this project is more focused on Information Visualization than Stream Clustering, further details regarding this topic will not be presented. However, a detailed survey can be found elsewhere [15].

\footnotetext{
${ }^{3}$ http://labs.sapo.pt

${ }^{4}$ https://reaction.fe.up.pt/socialbus
} 


\subsection{Information Visualization}

An important step in the data mining process is the interpretation of the results. It is often based on tools to visualize both the data and the knowledge extracted. The main properties that must be verified by these tools are: the displaying of the data and temporal behaviour; showing properties of the entire displayed scene and support interaction [8]. Visualization tools in a large multidisciplinary initiative require a pragmatic yet somewhat critical review of the ways visualization can be used to represent and to analyse data. In this project, the review of related work is focused on was done based on systems that collect data from Twitter.

For clustering visualization, one of the most common representations are graphs. The objects in each cluster are presented and the goal of assigning similar objects the shortest distance between clusters is maintained. Another option involves assigning different colors and objects to different concepts. For instance, overlapping ellipses over the most representative objects are displayed to represent similar objects. This approach was applied to study geographical lexical variation [7] and to classify events [4]. More recently, different clustering visualization methods were designed based on a different technique. For instance, clusters can be displayed in a more innovative and interactive way, enabling the user to easily identify content in each cluster [12].

Regarding visual representations on Twitter data, there are several approaches reported in the literature. TweetPos [17] is a web service that is intended to facilitate the analytical study of geographic tendencies in Twitter data feeds. In order to improve the user's experience with the tool, TweetPos relies on visual data structures such as heat maps and charts to represent the geo-spatial sources of tweets.

SensePlace2 [13] presents a geovisual analytics approach to support situational awareness for crisis events using Twitter. It focuses on leveraging explicit and implicit geographical information for tweets and on providing visual interface methods to enable understanding of place, time, and theme components of evolving situations.

TweetDrops [16] is a computer-based visualization tool designed for people who have not paid attention to sustainability in their life before. It opens up an opportunity for them to learn about energy conservation. It has two main visual components: the background rain drops, which represent the accumulation of energy related tweets collected from Twitter and the other is clickable foreground tweets with detailed content.

Earthquake [5] is a visualization tool that uses Twitter posts regarding the earthquake which occurred on the East Coast of the United States on August 23,2011 . It gathers information based on hashtags and displays the locations of different tweets in different time periods. It displays information as heat maps as well as graphs. 


\section{TweeProfiles3}

In order to obtain data from Twitter in real time, we integrated TweeProfiles2 in SocialBus. SocialBus is a platform that allows users to gather and store data from Twitter. Integrating SocialBus in TweeProfiles2 is a big improvement, due to the fact that we may restrict SocialBus to save only tweets with the required information for the task. Also, not only it allows TweeProfiles2 to be performed directly on the Twitter stream in real time, but also allows the information to be saved, in order to apply clustering to the same data. This is an important feature for evaluation of stream clustering algorithms.

\subsection{System Architecture}

TweeProfiles3 combines SocialBus' extraction and pre-processing methods to gather data from Twitter and the multidimensional stream clustering strategy from TweeProfiles2. The system architecture for TweeProfiles3 involves several modules, namely: Data Handling, Clustering, Data Mapping and Search.

Data Handling The design of the data collection software module separates webinterface logic from the background processes for data collection and storage. This is completely done by SocialBus who uses the open-source Twitter4J library for connection to the Streaming API and stores received tweets in MongoDB. The data collected suffers pre-processing tasks, namely time conversion and geodata filtering.

Clustering As the data is retrieved from MongoDB it is fed to the clustering algorithms used in TweeProfiles2: DenStream and DBSCAN. DenStream is responsible for the creation of micro-cluster that are then used as input to DBSCAN in order to create the macro-clusters.

Data mapping This module is responsible for data visualization. It displays the results of the clustering algorithms and of spatial, temporal and textual queries to the end users. The Google Maps ${ }^{5}$ and Leaflet ${ }^{6}$ Javascript APIs are used to display retrieved tweets and resulting clusters on a map.

Search Full-text search is incorporated in our systems to assist end users with textual analysis, speed-up queries on a large data-set and to produce a broader set of search results for each keyword specified by the user. Specifically, the system performs a match of the desired word to both tweets and set of words in all clusters. The system also incorporates a full spatial search enabling the users the define a specific region to analyse, as well as a temporal search so that the user can define a certain weekday to display all tweets and clusters and a timeline to go back in time 7 days.

\footnotetext{
${ }^{5}$ http://maps.google.com

${ }^{6}$ http://leaftletjs.com
} 


\subsection{Visualization System}

TweeProfiles3 exposes its functionality to end users through a php web application framework, entitled Codeigniter ${ }^{7}$. It also provides a simple web based data collection and retrieval interface, which is shown in Figure 1. All widgets are related in the sense that when selecting an element in the map, all other widgets update accordingly.

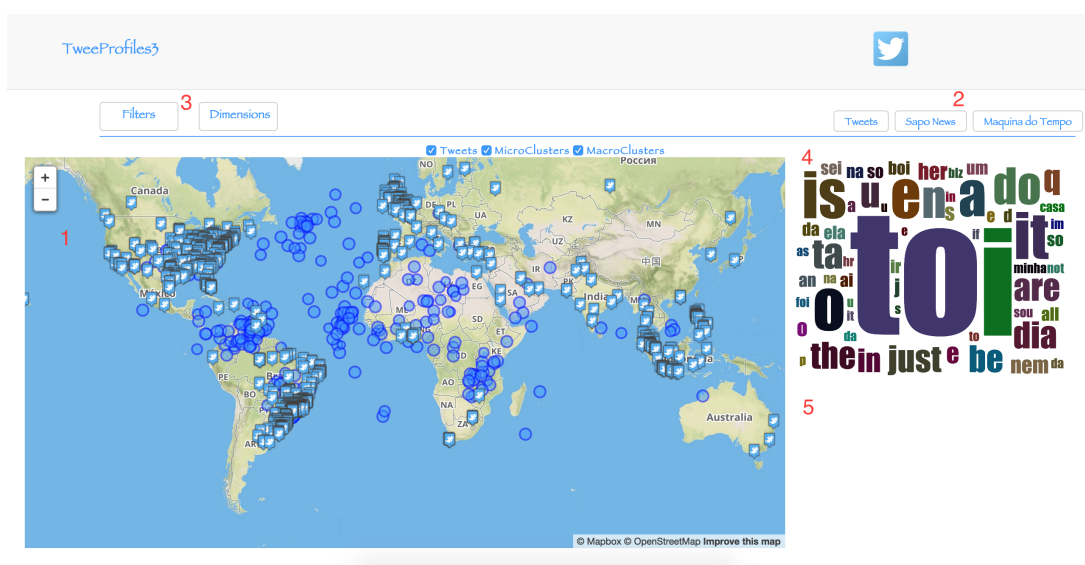

Fig. 1. TweeProfiles3's web interface.

The system interface includes 5 core components: 1) Tweet and cluster map; 2 ) tweet, news and entities list; 3) display/dimension controls; 4) wordcloud and 5) time graphics.

Tweet and Cluster map The map supports simultaneous tweets and clustering distribution overview. In one platform, a heatmap provides the overview for tweets and the clusters are displayed using simple markers. In a second platform, tweets' positions are displayed using markers and clusters with circles. Here we can get detailed information such as the number of words in the cluster, the creation time and position.

Tweet, News and Entities list The tweet list depicts the 1000 newest tweets for any query. The entity list displays any personality found in the processed tweets and the news list display the 20 more recent news. These were achieved by acessing public APIs from Labs Sapo: Verbetes to detect entities from the tweets and Máquina do Tempo, which provides the news per entity.

\footnotetext{
$\overline{7 \text { http://www }} \cdot$ codeigniter.com
} 
Display/Dimension controls These controls support query filtering with region selection, time range sliders (either choosing weekday or going back in time) and a minimalistic interface to specify terms for text-based queries. These controls also include a filter to specify the desired dimensions.

Wordcloud Displays the most frequent words in all clusters. The size of the words is equivalent to its frequency in tweets. The higher the frequency of said word, the bigger the word's size is. Clicking any word in the cloud performs a search for news related to that word in Sapo.pt.

Time graphics In this dimension we have two types of graphs. The first ones display the hour and weekday of the creation of all clusters (see Figure 2). They are represented by a circle with a center on the average time of all tweets and its size is directly dependent on the duration of the cluster. The second graph is inspired on a timeline using horizontal bars to characterize each cluster. The length of the bar is given by the earliest and latest timestamps of that cluster (see Figure 3).

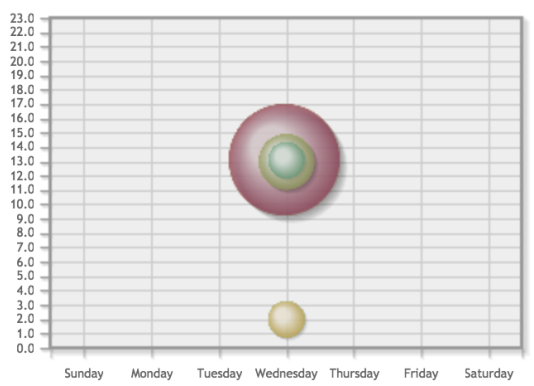

Fig. 2. Temporal visualization $-x-y$ graph.

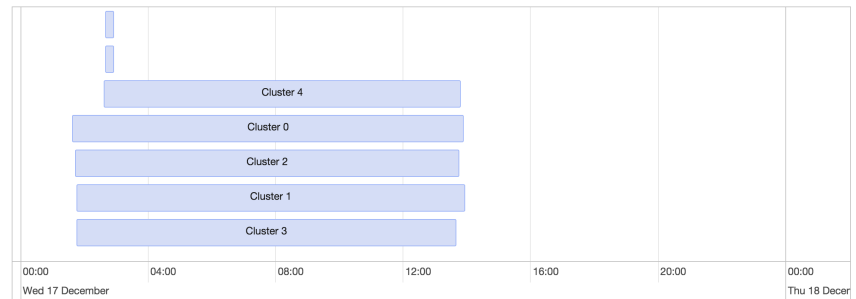

Fig. 3. Temporal visualization - timeline.

The chosen visualization strategy for the system assumes a spatio-temporal representation as the basis. This decision was taken based on the fact that time 
and space dimensions are the most intuitive and easily representable and interpretable. This representation approach was based on the methodology used by [7] but with two differences: the colors to represent each cluster and the shape of the clusters. One disadvantage of this type of representation is the overlapping markers and circles when zoomed out. The only solution for this problem is zooming in a specific region and/or using the available filters.

\section{Results}

The system was tested with a large scale data over a period of 30 days. The data collection, for testing purposes, was focused on tweets labelled in Portuguese, English, French and Spanish. All these tweets were retrieved without using any specific query method, besides the language filtering. The clustering algorithm parameters established are the same used in TweeProfiles2. Since the distance function weighting was not performed correctly in TweeProfiles2, we do not consider dimensional weighting in TweeProfiles3. Our goal is to provide a suitable interface for TweeProfiles2 multidimensional clustering process, and therefore we do not present the clustering results. A more extensive explanations of these results can be found elsewhere [14]. Furthermore, since we still lack an evaluation methodology suitable for the multidimensional nature of TweeProfiles, the results shown would have to be only examples of application and not a full-fledged evaluation. Therefore, we devote ourselves to the presentation of the user study used to validate our system and, in particular, the visualization tool.

\subsection{Usability Tests}

To understand if TweeProfiles3 met the desired features and design of end users, we performed an usability test. We asked the participants to use the tool to extract information from tweets, news and clusters, evaluating the time and detail of the result. These tests were made with $\mathrm{JPN}^{8}$ journalists, but unfortunately only 3 were available at the time of this publication.

The participants were asked to complete a form, for us to understand how they felt about the implemented features and the general use of TweeProfiles3. This survey was created based on [11] and people were able to evaluate, from 1-5, different features and aspects of the system ( 1 being that they disagreed with the statement and 5 that they fully agreed). Table 1 presents the questionnaire used for this user study, alongside the answer given by the three journalists.

We were able to understand that the users easily and effectively extracted information from both tweets and all news features. The ability to apply different filters to the data was highly appreciated and used, being the content filtering the one that got the most attention. All data shown on the map seemed to be useful for the tasks. An example given for the usage of tweet's details was the terrorist attack on Charlie Hebdo. The users stated that TweeProfiles3 would be

\footnotetext{
${ }^{8}$ http://jpn.up.pt/
} 
Table 1. User study questionnaire

\begin{tabular}{llll}
\hline Question & Journalist & 1 Journalist 2 & Journalist 3 \\
\hline Age & 25 & 23 & 27 \\
\hline Academic background & Bachelor & Bachelor & Masters \\
\hline Current position & Journalist & Journalist & Editor \\
\hline Years of experience in journalism & 3 & 3 & 4 \\
\hline System simplicity & 4 & 4 & 4 \\
\hline System efficiency for journalism? & 4 & 4 & 4 \\
\hline System understandability & 4 & 3 & 4 \\
\hline Information easily accessible & 5 & 3 & 4 \\
\hline System layout & 5 & 5 & 4 \\
\hline Tools available in the system & 4 & 3 & 4 \\
\hline General feedback & 4 & 4 & Map, News \\
\hline Most important features? & Map, News & Map, News & n/a \\
\hline Which tool would you remove? & n/a & Máquina do Tempo n/a \\
\hline Which feature needs improvement? & n/a & Graphics & Yes \\
\hline Are the connections to Sapo tools useful? Yes & Yes & News \\
\hline Most relevant Sapo tool used? & News & News & TP3 \\
\hline Do you consider the wordcloud useful? & Yes & Needs improvement Yes \\
\hline $\begin{array}{l}\text { Which is the most efficient method: } \\
\text { the tool (TP3) or traditional methods? }\end{array}$ & n/a & TP3 & \\
\hline
\end{tabular}

perfect to support an article regarding what happened, since they could not only gather information from the news, but also from what people were saying, giving the possibility to use such information in the article. Regarding the same topic, one aspect considered missing were hashtags and images. These two features are removed from the analysis and since the biggest trend from the attack included images and hashtags, a lot of information was lost. Improving the algorithm to use this would be a big step to TweeProfiles3.

One other aspect considered important by the journalists was the integration of Sapo platforms in TweeProfiles3. Even though the news list was more analysed than Máquina do Tempo, both were acknowledge as a plus.

At first people evaluated how simple the usage of the system was. All answers point to the fact that TweeProfiles3 is a simple system to use. This was our biggest concern when designing the application, to create a simple system with all the desired information. The users also agreed (with the same evaluation) that they were able to complete work effectively using TweeProfiles3. Regarding the usability of our system, one participant evaluated with a 4 , and one of them with a 3, on our 1-5 scale, how easy it was to understand. When it comes to the information we present, all respondents answered with 5 on how clear the information was organized on the screen, but regarding how easy it was to find the information they were searching, one person evaluated with a 3 , while others still evaluated with a 5 .

One other question all participants came to agreement was regarding the implemented features. All said that the most important were the map with all tweets/clusters and the news. However, they also considered that the time graphs and the wordcloud require improvement. When asked if they agreed with the integration of the platform from Sapo, all participants answered positively and also, all agreed that the news list was more relevant than Máquina do Tempo. 
When finally asking the participants if they were satisfied with the outcome of TweeProfiles3, the answers were positive, all rating 4, which means our system proved to be to the liking of all people who tested it. Even though there are some improvements to be made and some features to be added, and the number of journalists we had to test our application was fairly low, it was easy to understand how all participants considered TweeProfiles3 faster, more efficient and detailed than any other traditional method for gathering information. Platforms like this are seen as useful and necessary for the journalism community, and overall, TweeProfiles3 went in the right way, satisfying all participants.

\section{Conclusions}

In this project we developed an integration of a multidimensional Stream Clustering algorithm from TweeProfiles2 and SocialBus to create a visualization system capable of displaying the profiles found in all different dimensions. We also related these profiles with different news and entities from Sapo Labs and made the first attempt to evaluate the tool as a real-world application. The visualization tool made use of different visual patterns associated with all dimensions. Specifically, a map with information regarding both tweets and clusters, a timeline and a graph with timestamp details and a wordcloud with the most common words in all clusters. These widgets enable a simultaneous representation of the same information in different dimensions. Lastly, we performed a usability test with journalists to understand the efficiency, usability and overall performance of the platform. Despite the reduced number of interviewees, the results are promising. We have planned to tackle several problems in the future work: improve the data filtering and cleaning strategies, implement other widgets or filters that may lead to a more detailed analysis of the data, connect to external tools to support more languages and increase the amount of the journalists invited to the user study in order to provide our tests with statistical significance.

Acknowledgments. This work was partially supported by projects REACTION (Retrieval, Extraction and Aggregation Computing Technology for Integrating and Organizing News - UTA-Est/MAI/0006/2009), POPSTAR (Public Opinion and Sentiment Tracking, Analysis, and Research - PTDC/CPJCPO/116888/2010) and Sapo Labs. It was also financed by the ERDF - European Regional Development Fund through the Operational Programme for Competitiveness and Internationalisation - COMPETE 2020 Programme, and by National Funds through the FCT - Fundação para a Ciência e a Tecnologia (Portuguese Foundation for Science and Technology) within project POCI-010145-FEDER-006961.

\section{References}

1. Abreu, P.H., Amaro, H., Silva, D.C., Machado, P., Abreu, M.H.: Personalizing breast cancer patients with heterogeneous data. In: Proceedings of the 2013 IFMBE International Conference on Health Informatics, 2013. pp. 39-42 (2013) 
2. Abreu, P.H., Silva, D.C., Almeida, F., Mendes-Moreira, J.: Improving a Simulated Soccer Team's Performance Through a Memory-Based Collaborative Filtering Approach. Applied Soft Computing 23, 180-193 (2014)

3. Boanjak, M., Oliveira, E., Martins, J., Mendes Rodrigues, E., Sarmento, L.: Twitterecho: a distributed focused crawler to support open research with twitter data. In: Proceedings of the 21st international conference companion on World Wide Web. pp. 1233-1240. ACM (2012)

4. Boettcher, A., Lee, D.: Eventradar: A real-time local event detection scheme using twitter stream. In: Green Computing and Communications (GreenCom), 2012 IEEE International Conference on. pp. 358-367. IEEE (2012)

5. Crooks, A., Croitoru, A., Stefanidis, A., Radzikowski, J.: \# earthquake: Twitter as a distributed sensor system. Transactions in GIS 17(1), 124-147 (2013)

6. Cunha, T., Soares, C., Mendes Rodrigues, E.: Tweeprofiles: Detection of spatiotemporal patterns on twitter. In: Luo, X., Yu, J., Li, Z. (eds.) Advanced Data Mining and Applications, Lecture Notes in Computer Science, vol. 8933, pp. 123-136. Springer International Publishing (2014), http://dx.doi.org/10.1007/ 978-3-319-14717-8_10

7. Eisenstein, J., O'Connor, B., Smith, N.A., Xing, E.P.: A latent variable model for geographic lexical variation. In: Proceedings of the 2010 Conference on Empirical Methods in Natural Language Processing. pp. 1277-1287. Association for Computational Linguistics (2010)

8. Gahegan, M.: 11 visual exploration and explanation in geography analysis with light. Geographic Data Mining and Knowledge Discovery p. 291 (2009)

9. Jiawei, H., Kamber, M.: Data mining: concepts and techniques. San Francisco, CA, itd: Morgan Kaufmann 5 (2001)

10. Lee, C.H., Yang, H.C., Chien, T.F., Wen, W.S.: A novel approach for event detection by mining spatio-temporal information on microblogs. In: Advances in Social Networks Analysis and Mining (ASONAM), 2011 International Conference on. pp. 254-259. IEEE (2011)

11. Lewis, J.R.: Ibm computer usability satisfaction questionnaires: psychometric evaluation and instructions for use. International Journal of Human-Computer Interaction $7(1), 57-78$ (1995)

12. Liu, X., Hu, Y., North, S., Shen, H.W.: Compactmap: A mental map preserving visual interface for streaming text data. In: Big Data, 2013 IEEE International Conference on. pp. 48-55. IEEE (2013)

13. MacEachren, A.M., Jaiswal, A., Robinson, A.C., Pezanowski, S., Savelyev, A., Mitra, P., Zhang, X., Blanford, J.: Senseplace2: Geotwitter analytics support for situational awareness. In: Visual Analytics Science and Technology (VAST), 2011 IEEE Conference on. pp. 181-190. IEEE (2011)

14. Pereira, J.: TweeProfiles2: real-time detection of spatio-temporal patterns in Twitter. Master's thesis, Faculdade de Engenharia da Universidade do Porto (2014)

15. Silva, J.A., Faria, E.R., Barros, R.C., Hruschka, E.R., de Carvalho, A.C., Gama, J.: Data stream clustering: A survey. ACM Computing Surveys (CSUR) 46(1), 13 (2013)

16. Wang, X., Cosley, D.: Tweetdrops: a visualization to foster awareness and collective learning of sustainability. In: Proceedings of the companion publication of the 17th ACM conference on Computer supported cooperative work \& social computing. pp. 33-36. ACM (2014)

17. Wijnants, M., Blazejczak, A., Quax, P., Lamotte, W.: Tweetpos: A tool to study the geographic evolution of twitter topics. In: International Conference on Web Information Systems and Technologies. pp. 257-266 (2014) 